\title{
Estrategias de marketing con redes sociales en hoteles de cuatro y cinco estrellas en la ciudad de Tijuana, Baja California
}

\author{
Ana María Miranda Zavala* \\ Isaac Cruz Estrada \\ María Ramona Valle Ascencio \\ Juan Carlos Flores Trejo \\ Universidad Autónoma de Baja California
}

\section{Resumen}

El presente artículo tiene como objetivo identificar las estrategias de marketing con redes sociales que realizan los hoteles de cuatro y cinco estrellas de la ciudad de Tijuana, Baja California, México, así como revisar sus características y contenidos, reconociendo su importancia como medio de comunicación con los clientes y la necesidad de ser analizadas periódicamente al igual que sus resultados. Con ese propósito se caracterizaron los atributos que los clientes de los hoteles consideran esenciales y que aumentan su confianza en estos medios. Para ello se efectuó un análisis cualitativo de las redes sociales de 16 hoteles de cuatro y cinco estrellas de Tijuana, registrados en la Secretaría de Turismo del Estado (Secture) y en la Asociación de Hoteles del Noroeste A.C. (Ashono). Asimismo, se aplicó una encuesta a 267 huéspedes con el fin de determinar los atributos que habrían de elevar la eficiencia y pertinencia de los resultados de esta estrategia de marketing..

\section{Palabras clave}

Estrategias de marketing, redes sociales, hoteles, turistas, comunicación.

*Correo electrónico: amiranda@uabc.edu.mx, icruz@uabc.edu.mx, marvalle@uabc.edu.mx,jcft@uabc.edu.mx

Recibido: 30/03/2014 · Aceptado: 25/04/2014 


\title{
Social Network marketing strategies of four and five star hotels in the city of Tijuana, Baja California
}

\author{
Ana María Miranda Zavala* \\ Isaac Cruz Estrada \\ María Ramona Valle Ascencio \\ Juan Carlos Flores Trejo \\ Universidad Autónoma de Baja California
}

\begin{abstract}
The goal of this article is to identify the social network marketing strategies performed by four and five stars hotels in the city of Tijuana, Baja California, Mexico reviewing their features and contents. The importance of social networks as a means of communication with customers as well as the need for continuous upgrading and evaluation has to be recognized. This will allow hotels to increase the efficiency of these tools by identifying areas of opportunity that might have been overlooked. With this goal in mind, the features of social media considered by customers as essential and trust providing were characterized by means of a qualitative analysis of the social network content of 16 four and five star hotels registered with the Department of Tourism (Secture) and the Northwest Hotel Association (Ashono). A survey was applied to 267 hotel guests in order to determine the features and contents that would increase the efficiency and pertinence of this social media communication strategy.
\end{abstract}

\section{KEY WORDS}

Marketing strategies, social networks, hotels, tourists, communication

*E-mail: amiranda@uabc.edu.mx, icruz@uabc.edu.mx,marvalle@uabc.edu.mx, jcft@uabc.edu.mx 


\section{Introducción}

Las redes sociales en internet se han manifestado como una de las formas más generalizadas y revolucionarias de crear lazos y comunidades virtuales; por su alcance y por las posibilidades de desarrollo, la mercadotecnia ha visto en ellas un potencial para implementar las estrategias indispensables para acercarse al mercado y buscar nuevas oportunidades de negocio. Conde et al. (2011) señalan la necesidad de aprovechar el comercio electrónico en el ámbito turístico para mejorar las operaciones tradicionales practicadas, tanto en la estrategia empresarial como en la reingeniería del negocio turístico. Estas herramientas pueden ayudar a hacer más eficientes los procesos de gestión y apoyar la toma de decisiones para que las organizaciones actúen con mayor efectividad.

Conde et al. (2011) destacan, además, que el turismo electrónico se ha desarrollado principalmente por las siguientes razones: por demandas de la nueva sociedad del ocio y la tecnología, por la situación favorable introducida por el comercio electrónico, por la exigencia de mejorar el sector turístico y por la entrada de los nuevos modelos de gestión basados en la satisfacción del cliente.

Por lo tanto, reconocer las estrategias de marketing en redes sociales que llevan a cabo los hoteles de cuatro y cinco estrellas de Tijuana, Baja California, México, ipermitirá aportar conocimiento para incrementar su eficiencia?

\section{Objetivos de la investigación}

\section{Objetivo general de la investigación}

Identificar las estrategias de marketing en redes sociales que realizan los hoteles de cuatro y cinco estrellas de la ciudad de Tijuana.

\section{Objetivos específicos}

- Analizar el comportamiento de los hoteles de cuatro y cinco estrellas de Tijuana y caracterizar las tácticas empleadas en sus estrategias de marketing en redes sociales.

- Caracterizar las redes sociales utilizadas por los clientes de los hoteles. 
- Determinar los atributos de los contenidos de las redes sociales del hotel que los clientes consideran indispensables y que los incitan a servirse de estos sitios en internet.

\section{Antecedentes}

De acuerdo con Casaló, Flavián y Guinalíu (2012), desde el punto de vista de la mercadotecnia, las redes sociales promovidas por las organizaciones poseen carácter comercial, lo que ha llevado a las empresas a aprovechar las bondades de estas herramientas que les permiten conocer más a los usuarios, reducir sus costos del servicio de atención al cliente y mejorar sus investigaciones de mercado, con la intención de involucrar a los consumidores en el diseño de nuevos productos.

\section{Redes sociales en el entorno global}

García (2009) afirma que las redes sociales son sin lugar a duda una de las mayores innovaciones de la Web 2.0. Construidas con el propósito de interconectar a los usuarios que comparten aficiones, amistades, ideas, fotografías, videos y elementos multimedia de todo tipo (atribuyéndose una buena parte del tráfico de información en la red mundial de internet), estas herramientas se están volviendo esenciales en la vida cotidiana de las personas.

La actividad en las redes sociales ha ido creciendo en el mundo, y los patrones de uso varían dependiendo de la región y el país. Según eMarketer (2012), los visitantes de Asia y el Pacífico predominan en estos sitios de internet. En la gráfica 1 se muestra la tendencia en el uso de estos medios por regiones del mundo.

Para Hütt (2012), las redes sociales se aplican en todas las áreas de las organizaciones, entre las que destacan la medicina, la educación, la gestión empresarial, la mercadotecnia y el turismo; de este modo, debido al auge de estas tecnologías en todo el mundo, las compañías han decidido invertir en ellas para incorporarlas a sus estrategias de marketing, ya que las posibilidades de interacción con sus públicos son muy amplias y van desde contar con un canal de retroalimentación hasta con uno de promoción, información y mercadeo. 


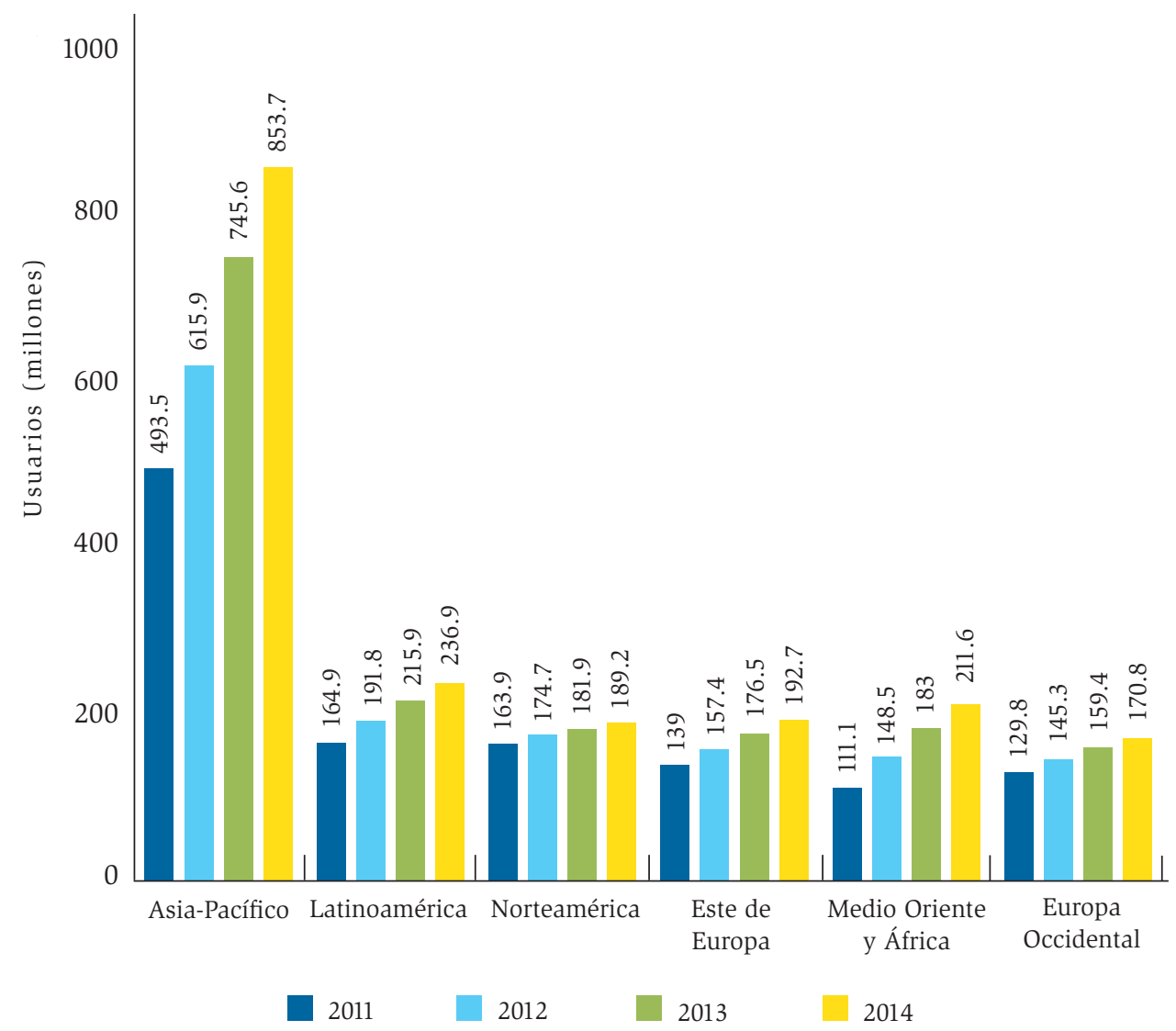

Fuente: Elaboración propia a partir de eMarketer (2012).

Gráfica 1. Tendencia en el uso de redes sociales por regiones en el mundo (en millones de usuarios)

Asimismo, existe la opción de contar con perfiles a los cuales los usuarios pueden sumarse como seguidores, lo que permite establecer una dinámica menos invasiva y más enfocada hacia sus necesidades y expectativas. 


\section{Redes sociales en México}

La Asociación Mexicana de Internet (AMIPCI) afirma que en 2012 aumentó en México el uso de redes sociales: la edad promedio de los internautas mexicanos oscila entre los 12 y los 24 años de edad; los jóvenes de este país consumen un tiempo considerable en estos medios, y sus primordiales acciones son enviar y recibir correos electrónicos. La gráfica 2 expone las principales actividades: sobresale que $80 \%$ de los usuarios utiliza este medio para enviar y recibir correos electrónicos, 77 \% para acceder a redes sociales, $71 \%$ para buscar información, $55 \%$ para enviar y recibir mensajes instantáneos, $44 \%$ para efectuar operaciones de banca en línea y solo 29 \% para hacer compras en línea.

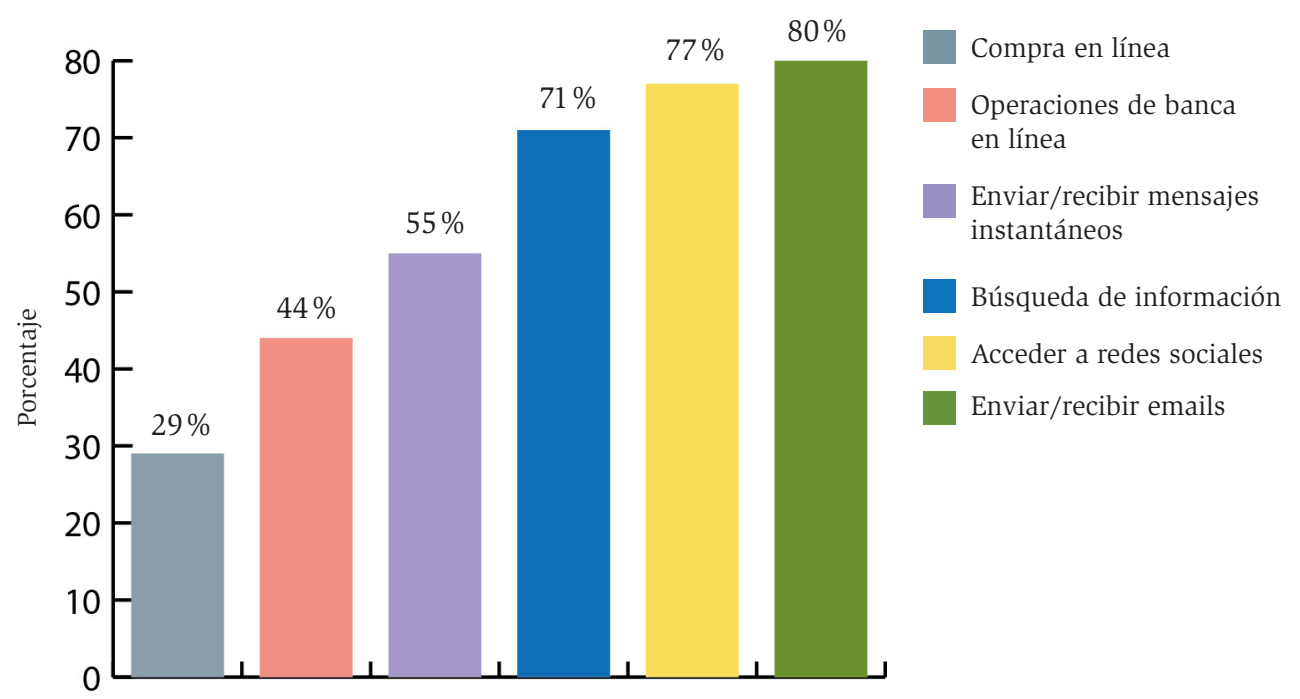

Fuente: Elaboración propia a partir de AmIPCI (2012).

Gráfica 2. Principales actividades online en México 
Las herramientas de internet están cambiando la forma de comunicarse entre las personas, cada día se eleva el número de usuarios que se registran en alguna red social. En México, los internautas pertenecen en promedio a cuatro redes sociales. La gráfica 3 expone las preferidas por los mexicanos: Facebook sobresale con el acceso diario de $90 \%$ de los usuarios; después YouTube, con $77 \%$; $61 \%$ entran diario a Twitter; $44 \%$ a Google +, y solo $10 \%$ a Hi5. En cuanto a la inscripción a estos medios, se tiene que 90 \% está en Facebook, 60 \% en YouTube, $55 \%$ en Twitter, $34 \%$ en Google + y solo $25 \%$ en Hi5.

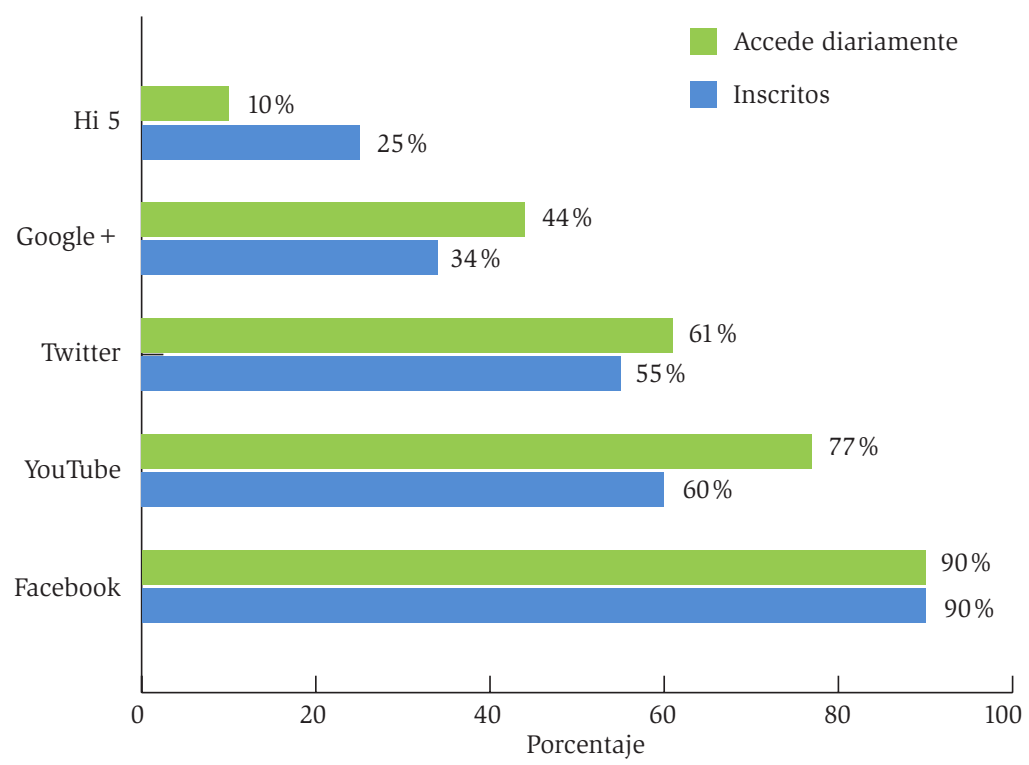

Fuente: Elaboración propia a partir de AmIPCI (2012).

Gráfica 3. Redes sociales más usadas en México

Hütt (2012) señala que las empresas han incrementado su publicidad en las redes digitales para dar a conocer sus marcas o servicios, pues este tipo de espacios permite analizar el comportamiento del usuario a través de variables como las compras en línea o la emisión de comentarios específicos. Asimismo, estas plataformas sirven para ofrecer servicios de atención al cliente, a causa de la facilidad para interactuar con los usuarios. 


\section{Marketing con redes sociales}

Según Kotler y Armstrong (2003), el marketing es un proceso social y administrativo por el que los individuos obtienen lo que necesitan y desean, mediante la creación y el intercambio de productos y de valor con otros; es el arte de atraer, conservar y cultivar clientes rentables.

Desde el punto de vista social, Baena (2011) asevera que se trata de un proceso mediante el cual las personas consiguen lo que necesitan y demandan por medio de la oferta y el libre intercambio de productos o servicios con otros individuos; para que esto se lleve a cabo es indispensable que cada una de las partes tenga algo de valor para la otra.

Ahora bien, para Pérez (2010), el marketing en las redes sociales es un mecanismo muy efectivo para conocer la opinión de los clientes sobre los servicios de la empresa, también permite exponerla e incrementar el flujo de la publicidad, por lo que es clave lograr su eficiencia para que el cliente reconozca el trabajo y la dinámica en que la organización se ocupa en satisfacer sus necesidades, de lo contrario puede ser una bomba inmediata que desacredite el esfuerzo realizado.

Laudon y Guercio (2009), por su parte, consideran que el marketing en internet es muy diferente del marketing común por la naturaleza de sus medios y capacidades. El objetivo del primero es crear relaciones con los clientes, identificar sus necesidades, con la finalidad de que las organizaciones obtengan rendimientos mayores al promedio. En el cuadro 1 se ofrecen los planteamientos de algunos autores acerca de las ventajas de las redes sociales en la mercadotecnia de la empresa.

Cuadro 1. Marketing en redes sociales

\begin{tabular}{ll} 
Autor & VENTAJAS DEL USO DE LAS REDES SOCIALES EN LA MERCADOTECNIA \\
Morales (2010) & Mejora la comunicación con los clientes \\
Colvée (2007) & $\begin{array}{l}\text { Aumenta la comunicación con los clientes } \\
\text { Pérez (2010) }\end{array}$ \\
$\begin{array}{l}\text { Conocer la opinión de los clientes e incrementa } \\
\text { el flujo de la publicidad }\end{array}$ \\
Maqueira \& Bruque (2009) & $\begin{array}{l}\text { Fortalece la comunicación entre la empresa } \\
\text { y el consumidor }\end{array}$ \\
\hline
\end{tabular}

Fuente: Elaboración propia. Morales (2010), Colvée (2007), Pérez (2010), Maqueira \& Bruque (2009) 
Como se puede apreciar, Morales, Colvée, Pérez y Maqueira y Bruque coinciden en que el marketing en redes sociales mejora y fortalece la comunicación con los clientes. Pérez, además, sostiene que aumenta el flujo de la publicidad.

\section{Marketing con redes sociales en el sector turístico}

En la actualidad, las empresas turísticas se encuentran sumergidas en un mercado muy competitivo y en una difícil situación económica, debido a la incesante variación del entorno globalizado; las actitudes de los consumidores cambian constantemente y ahora esperan conseguir más valor por su dinero, por lo que la prioridad de las empresas ha sido retener a sus clientes en lugar de captar adicionales, considerando que conseguir un nuevo cliente es cinco veces más caro que mantener uno actual (Benavides, 2012).

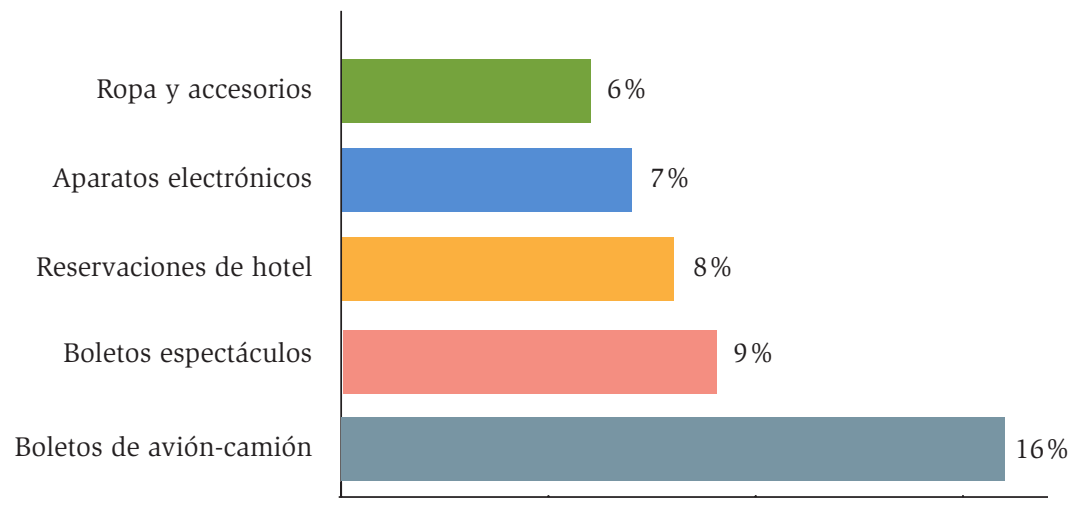

Fuente: Elaboración propia a partir de AmIPCI (2011).

\section{Gráfica 4. Ventas de unidades por Internet}

Ahora bien, las empresas del sector turístico, en específico las del área de hotelería, tienen grandes posibilidades para conquistar al turista a través de las experiencias compartidas por sus propios clientes en las redes sociales. Gallego (2008) expone el ejemplo de las agencias de viajes, que están buscando novedosas formas de llegar a su mercado, siendo que los clientes utilizan internet para realizar reservaciones de los diferentes servicios. 
Nielsen (2012) destaca que, si bien la confianza de los usuarios en la publicidad en línea está aumentando, $92 \%$ de los consumidores del mundo confía más en la publicidad de boca en boca (recomendaciones hechas por amigos y familiares, y en segundo lugar los comentarios que publican los consumidores en la web), aunque $70 \%$ de los encuestados por internet cree en esta fuente de información.

Del mismo modo, en México el sector turismo se encuentra posicionado en los primeros lugares de ventas por unidades en internet; la gráfica 4 describe los datos recopilados por la Amirci y el porcentaje de ventas hasta 2011.

De acuerdo con la gráfica 4, las ventas de boletos de avión o transporte terrestre alcanzaron $16 \%, 9 \%$ corresponde a ventas de boletos para espectáculos, y $8 \%$ a las reservaciones en línea. En la figura 1 se desglosan las principales redes sociales utilizadas por las empresas en México:

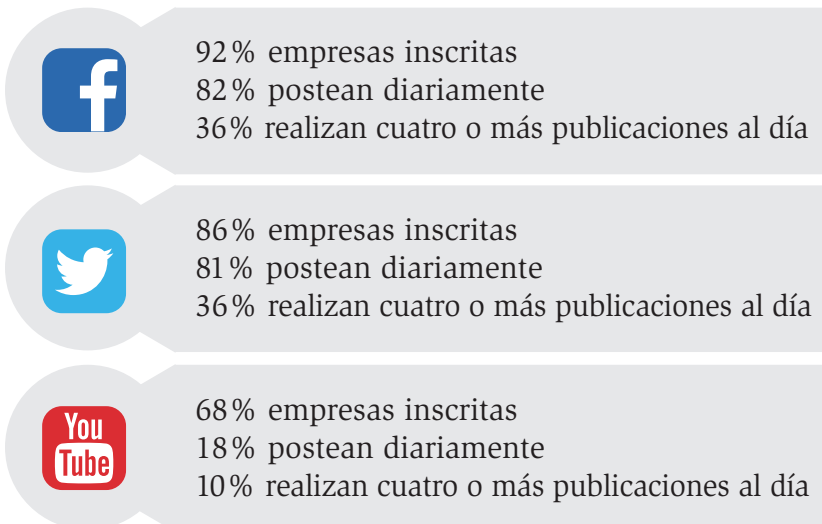

Fuente: Elaboración propia a partir de AMIPCI (2012).

Figura 1. Principales redes sociales en empresas mexicanas

Facebook es la red social con mayor presencia en las organizaciones mexicanas, sin embargo cabría analizar cuáles son las más apropiadas para el sector.

Domínguez y Araújo (2012) aseveran que internet es un elemento clave para el sector turístico. En este sentido puede destacarse que hoy en día el turista 
ya no solo tiene el poder adquisitivo, sino también la posibilidad de planificar, gestionar y, si no es de su agrado, compartir la experiencia recibida con todo aquel que tenga acceso a los medios electrónicos; el boca-oído ahora tiene dimensiones incalculables, lo cual ha llevado a organismos y a empresas a visualizar esta oportunidad de mercado que en breve puede convertirse en una gerencia básica; lo que ahora genera una ventaja, dentro de poco tiempo será una demanda obligatoria del usuario.

\section{Metodología}

Para realizar el presente estudio se analizó el comportamiento de 16 hoteles de cuatro y cinco estrellas de la ciudad de Tijuana, registrados en la Secretaría de Turismo del Estado (Secture) y en la Asociación de Hoteles del Noroeste (Ashono), revisando su actividad en Facebook, Twitter y TripAdvisor. El cuadro 2 señala los factores para el estudio de los atributos considerados en las redes sociales de los hoteles.

Otra parte de la investigación consistió en el análisis descriptivo de las redes sociales que utilizan los clientes de los hoteles de cuatro y cinco estrellas de Tijuana, así como los elementos que juzgan indispensables en estos sitios y que son fundamentales para que recurran a estas herramientas en internet.

La aplicación de las técnicas cualitativa y cuantitativa se llevó a cabo del 1 de junio al 1 de agosto de 2013.

\section{Población objetivo}

La población objetivo fueron 16 hoteles de cuatro y cinco estrellas de la ciudad de Tijuana, registrados por la Secture y por Ashono en 2013.

Para el análisis descriptivo que define los atributos esenciales para los visitantes, se tomó como población objetivo a los clientes de los hoteles de cuatro y cinco estrellas. Se estima un total de 314836 huéspedes, calculado por el número total de habitaciones de cada hotel con una ocupación de 100 por ciento en el mes de julio de 2012, ya que -según los indicadores obtenidos en el Sistema Nacional de la Información Estadística del Sector Turismo de México (Datatur)el comportamiento de este factor en este tipo de empresas es similar. 
Cuadro 2. Factores de Internet que influyen en la decisión de compra

\begin{tabular}{|c|c|c|c|}
\hline & \multicolumn{3}{|r|}{ FACTORES } \\
\hline \multirow[t]{6}{*}{$\begin{array}{l}\text { Izquierdo y } \\
\text { Martínez (2009) }\end{array}$} & \multirow[t]{5}{*}{ Confianza } & \multicolumn{2}{|c|}{$\begin{array}{l}\text { Señal: precio, marca, inversión en comunicación, servicio } \\
\text { postventa, devolución del dinero y garantía por posibles con- } \\
\text { tingencias }\end{array}$} \\
\hline & & \multicolumn{2}{|c|}{$\begin{array}{l}\text { Confianza hacia la empresa: habilidad, benevolencia e inte- } \\
\text { gridad }\end{array}$} \\
\hline & & \multicolumn{2}{|c|}{ Confianza hacia internet: privacidad y seguridad } \\
\hline & & \multicolumn{2}{|c|}{$\begin{array}{l}\text { Costos de transacción: costos, incertidumbre y activos } \\
\text { específicos }\end{array}$} \\
\hline & & \multicolumn{2}{|c|}{$\begin{array}{l}\text { Riesgo: riesgo funcional, financiero, físico, social, psicológico } \\
\text { y temporal }\end{array}$} \\
\hline & \multicolumn{3}{|c|}{ Gestión de la información } \\
\hline \multirow{4}{*}{$\begin{array}{l}\text { Ruíz y Sanz } \\
\text { (2006) }\end{array}$} & \multirow[t]{4}{*}{ Motivación } & \multicolumn{2}{|l|}{ Tiempo } \\
\hline & & \multicolumn{2}{|c|}{ Comodidad: volumen de compra } \\
\hline & & \multicolumn{2}{|l|}{ Precio } \\
\hline & & \multicolumn{2}{|c|}{ Amplitud de surtido } \\
\hline \multirow{13}{*}{$\begin{array}{l}\text { Martínez, Bernal } \\
\text { y Mellinas (2012) }\end{array}$} & \multicolumn{3}{|c|}{ Número de estrellas } \\
\hline & \multicolumn{3}{|c|}{ Número de fotos en la web } \\
\hline & \multicolumn{3}{|c|}{ Número de videos en la web } \\
\hline & \multicolumn{2}{|l|}{ Tipo de web } & $\begin{array}{l}\text { Web propia, web integrada en la de una } \\
\text { cadena hotelera }\end{array}$ \\
\hline & \multicolumn{2}{|c|}{ Motor de reserva propios } & $\begin{array}{l}\text { No tiene motor propio cuando lo que incluye } \\
\text { es un enlace a booking.com }\end{array}$ \\
\hline & \multicolumn{2}{|l|}{ Q de calidad } & Indicación en la página web \\
\hline & \multicolumn{2}{|c|}{ Servicio Wi-Fi } & Indicación en la página \\
\hline & \multicolumn{2}{|c|}{$\begin{array}{l}\text { Vinculación con cadenas } \\
\text { hoteleras }\end{array}$} & $\begin{array}{l}\text { Independientes, vinculado a pequeña cadena, } \\
\text { vinculado a gran cadena }\end{array}$ \\
\hline & \multicolumn{2}{|c|}{$\begin{array}{l}\text { Uso principales redes so- } \\
\text { ciales }\end{array}$} & \\
\hline & \multicolumn{2}{|c|}{ Registrado en Google Maps } & \\
\hline & \multicolumn{2}{|l|}{ Población } & \\
\hline & \multicolumn{2}{|l|}{ Booking } & Numero de críticas, puntuación media \\
\hline & \multicolumn{2}{|l|}{ Tripadvisor } & Numero de críticas, puntuación media \\
\hline \multirow[t]{4}{*}{$\begin{array}{l}\text { Rojas, Arango } \\
\text { y Gallego (2009) }\end{array}$} & \multirow[t]{4}{*}{ Confianza } & \multicolumn{2}{|c|}{$\begin{array}{l}\text { Aspectos de diseño en la web: disponibilidad, diseño } \\
\text { atractivo y estructura de la información en la web, } \\
\text { sensación de comodidad. }\end{array}$} \\
\hline & & \multicolumn{2}{|c|}{ Tiempo de respuesta } \\
\hline & & Beneficios & ociales \\
\hline & & Invasión d & la privacidad \\
\hline
\end{tabular}

Fuente: Elaboración propia. a partir de Izquierdo y Martínez (2009), Ruíz y Sanz (2006), Martínez,

Bernal y Mellinas (2012), Rojas, Arango y Gallego (2009). 
Cuadro 3. Hoteles de cuatro y cinco estrellas que conforman las unidades de muestreo de la investigación

\begin{tabular}{|rll} 
& Nombre DEL HOTEL & NúMERo DE ESTRELLAS \\
\hline 1 & City Junior & Cuatro \\
2 & City Express & Cuatro \\
3 & Fiesta Inn Tijuana Otay & Cuatro \\
4 & Holiday Inn & Cuatro \\
5 & Hacienda del Río & Cuatro \\
6 & La Mesa Inn & Cuatro \\
7 & Lausana & Cuatro \\
8 & Real del Río & Cuatro \\
9 & Principado & Cuatro \\
10 & Grand Hotel Tijuana & Cinco \\
11 & Camino Real Tijuana & Cinco \\
12 & Lucerna & Cinco \\
13 & Marriott Tijuana & Cinco \\
14 & Palacio Azteca & Cinco \\
15 & Pueblo Amigo Inn & Cinco \\
16 & Real del Mar & Cinco \\
\hline
\end{tabular}

Fuente: Elaboración propia.

Asimismo, de acuerdo con Fischer y Navarro (2000), para poblaciones finitas (menos de 500000 elementos), como la de la investigación realizada (314 836 huéspedes), se utiliza la siguiente fórmula.

$$
n=\frac{\sigma^{2} N p q}{\mathrm{e}^{2}(N-1)+\sigma^{2} p q}
$$

En dónde:

$\boldsymbol{\sigma}^{2}=$ Corresponde al nivel de confianza seleccionado (95\%).

$\mathrm{N}$ = Universo o población.

$\mathrm{p}$ = probabilidad a favor (cuando no se conoce en la población, siempre se asume que es de 0.5).

$\mathrm{q}=$ probabilidad en contra. 
e $=$ Error de estimación a permitir $( \pm 6 \%)$.

$\mathrm{n}=$ Tamaño de la muestra.

$$
n=\frac{\left(1.96^{2}\right) * 314836 * 0.5 * 0.5}{0.06^{2} *[314836-1]+\left[1.96^{2} * 0.5 * 0.5\right]}=\frac{302368.4944}{1134.3664}=266.55=267
$$

Por lo tanto, se aplicaron 267 encuestas.

\section{Distribución de la muestra para la encuesta}

El instrumento se aplicó de manera proporcional en los hoteles de cuatro y cinco estrellas, considerando una ocupación de 100 por ciento en julio de 2013 y seleccionando a los huéspedes por habitación. En el cuadro 4 se presenta la distribución de las encuestas:

Cuadro 4. Número de encuestas aplicadas por cada unidad de muestreo de la investigación

\begin{tabular}{|clcc|}
\hline Núm. & Nombre DEL HOTEL & NúM. DE HABITACIONES & NúM. ENCUESTAS \\
\hline 1 & City Junior & 134 & 14 \\
\hline 2 & City Express & 127 & 13 \\
\hline 3 & Fiesta Inn Tijuana Otay & 142 & 15 \\
\hline 4 & Holiday Inn & 127 & 13 \\
\hline 5 & Hacienda del Río & 131 & 14 \\
\hline 6 & La Mesa Inn & 139 & 15 \\
\hline 7 & Lausana & 140 & 15 \\
\hline 8 & Real del Río & 105 & 11 \\
\hline 9 & Principado & 49 & 5 \\
10 & Grand Hotel Tijuana & 422 & 44 \\
\hline 11 & Camino Real Tijuana & 263 & 28 \\
\hline 12 & Lucerna & 168 & 18 \\
\hline 13 & Marriott Tijuana & 209 & 22 \\
\hline 14 & Palacio Azteca & 201 & 21 \\
\hline 15 & Pueblo Amigo Inn & 106 & 11 \\
\hline 16 & Real del Mar & 76 & 8 \\
\hline & & 2539 & 267 \\
\hline
\end{tabular}




\section{Confiabilidad}

Para la confiabilidad del instrumento se utilizó el alpha de Cronbach, cuyo resultado se presenta en el cuadro 5 .

Cuadro 5. Análisis de confiabilidad Alpha de Cronbach
\begin{tabular}{|l|l|}
\hline CONCEPTO & Alpha de Cronbach \\
\hline CuESTIONARIO & 0.895 \\
\hline
\end{tabular}

Fuente: Elaboración propia.

Según Hernández, Fernández y Baptista (2006), un resultado de 0.25 en la correlación o coeficiente indica baja confiabilidad; 0.50 significa media o regular; mayor a 0.75 es aceptable, y mayor a 0.90 es elevada. Por lo tanto, los datos obtenidos (0.895) revelan una confiabilidad aceptable.

\section{Resultados del estudio cualitativo}

Se realizó un análisis cualitativo del 1 de junio al 1 de agosto de 2013 de cada red social de los hoteles de cuatro y cinco estrellas de Tijuana. En particular se encontró que $62.5 \%$ efectúa actualizaciones constantes de las promociones, pero la mayoría son de los servicios del restaurante del hotel. En cuanto a las fotografías y videos de las instalaciones, se observó que solo $12.5 \%$ de las redes sociales muestran los diversos espacios físicos y servicios que se ofrecen, y las fotografías están organizadas por categorías o secciones en el sitio web; el resto no las tiene organizadas y la mayoría son ajenas a los servicios del hotel (como fotos de los empleados).

Se envió el siguiente mensaje a los hoteles por medio de las redes sociales: “¿Podría por favor, enviarme información de las habitaciones y sus precios, al correo miranda_miza@hotmail.com? Gracias de antemano”. Solo 31.3 \% de los hoteles lo respondieron, en un tiempo promedio de cinco días, como muestra el cuadro 6 . 
Cuadro 6. Registro de mensajes enviados por la red social al hotel

\begin{tabular}{rcc} 
& \multicolumn{2}{c}{ FECHA DEL MENSAJE } \\
\hline Núm & Enviado & Respuesta \\
1 & $08 / 07 / 2013$ & No \\
2 & $08 / 07 / 2013$ & No \\
3 & $08 / 07 / 2013$ & No \\
4 & $08 / 07 / 2013$ & No \\
\hline 5 & $09 / 07 / 2013$ & No \\
\hline 6 & $09 / 07 / 2013$ & No \\
7 & $09 / 07 / 2013$ & No \\
\hline 8 & $09 / 07 / 2013$ & No \\
\hline 9 & $09 / 07 / 2013$ & $10 / 07 / 2013$ \\
10 & $09 / 07 / 2013$ & $11 / 07 / 2013$ \\
11 & $10 / 07 / 2013$ & $12 / 07 / 2013$ \\
12 & $12 / 07 / 2013$ & $22 / 07 / 2013$ \\
13 & $09 / 07 / 2013$ & No \\
14 & $10 / 07 / 2013$ & No \\
15 & $10 / 07 / 2013$ & No \\
16 & $09 / 07 / 2013$ & $17 / 07 / 2013$ \\
\hline
\end{tabular}

Fuente: Elaboración propia.

\section{Resultados del estudio cuantitativo}

En la gráfica 5 se muestra que $86.8 \%$ de los clientes acceden a Facebook para obtener información del hotel en el que piensan hospedarse, $7.7 \%$ utiliza Twitter y solo $5.5 \%$ TripAdvisor.

En la gráfica 6 se describen los atributos que los huéspedes encuestados manifestaron deben estar presentes en la red social del hotel; en la categoría de muy importante sobresalen datos sobre la ubicación (60.8\%); los precios (42.7\%); información de las promociones (41\%); el servicio de restaurante $(35.2 \%)$; número de estrellas (29.5\%); videos del hotel (27.8\%); fotografías del hotel (24.2\%), e información acerca del servicio Wi-Fi (23.8\%). 


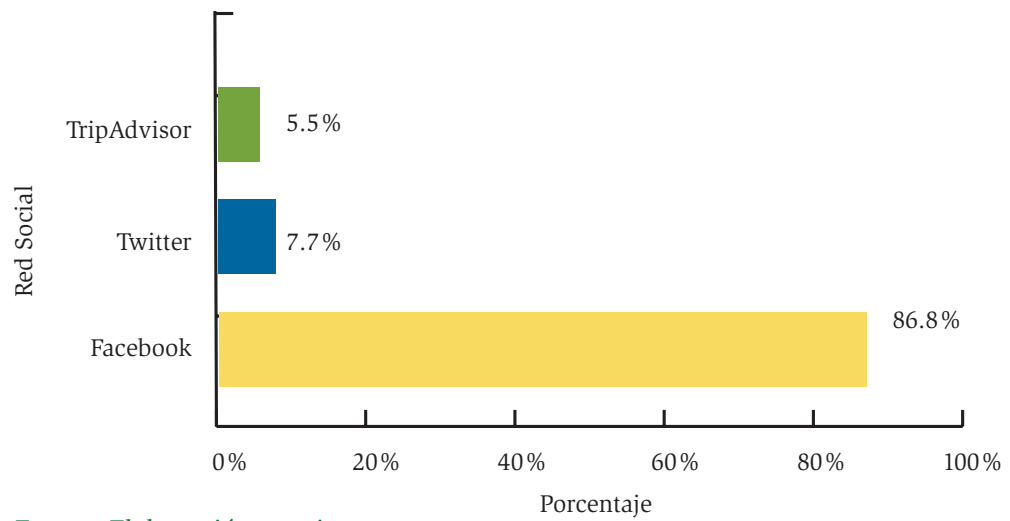

Fuente: Elaboración propia.

Gráfica 5. Red social que utilizan para obtener información del hotel.

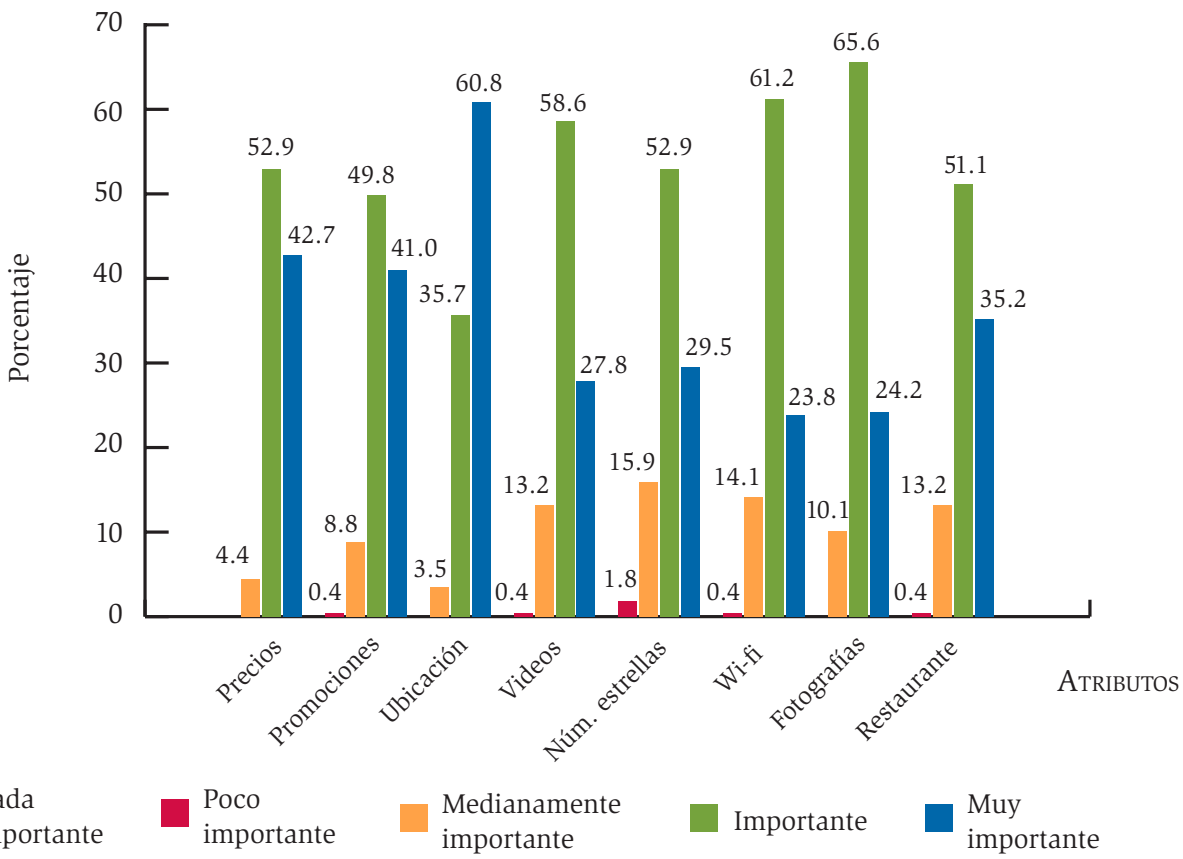

Fuente: Elaboración propia. 
En la gráfica 7 se presentan los factores que aumentarían la confianza de los encuestados en el uso de las redes sociales del hotel: en la categoría de muy importante destacan la actualización de contenidos (64.3\%); la calidad de los contenidos $(61.7 \%)$; la rapidez de respuesta a las preguntas realizadas en la red social (59\%); que el hotel respete las promociones publicadas (41.9\%); los servicios disponibles (22.9\%); la publicación de las áreas del hotel (21.1\%); que incluya enlaces a información turística (18.9\%) y que tenga enlaces a otros sitios web $(14.5 \%)$.

De acuerdo con los hallazgos encontrados en la investigación, los atributos que los hoteles deben incluir en una red social son: precios, promociones, ubicación, videos, número de estrellas, Wi-Fi, fotografías y servicio de restaurante. Asimismo, en el estudio "Los hoteles de la región de Murcia ante las redes sociales y la reputación online”, Martínez, Bernal y Mellinas (2012) sostienen que los atributos para la red social son: el número de estrellas, de fotos en la web y de videos, así como servicio Wi-Fi, que esté registrado en Google Maps y que se vincule con cadenas hoteleras. Lo cual coincide con la mayoría de los elementos que debe tener una red social del hotel.

\section{Comentario final}

Del presente proyecto se desglosan resultados relevantes para los objetivos planteados en esta investigación sobre el comportamiento de los hoteles de cuatro y cinco estrellas en la ciudad de Tijuana, y que caracterizan las tácticas empleadas en la utilización de las estrategias de marketing en las redes sociales.

Se concluye que hasta $62.5 \%$ de los hoteles realiza actualizaciones constantes de las promociones; sin embargo, para que los clientes aumenten su confianza en estos medios, deben respetarse tales publicaciones y hacer efectivas las promociones establecidas para incrementar la relación con los turistas que acceden a este servicio y, en consecuencia, influir en su decisión de compra.

También debe diversificarse el tipo de actualizaciones realizadas, ya que la mayoría son con referencia al servicio de restaurante, cuando existen otras categorías relevantes descritas por los clientes, como la calidad de los contenidos publicados, la rapidez de las respuestas a sus preguntas, además de videos y 
Estrategias de marketing con redes sociales en hoteles de cuatro y cinco estrellas

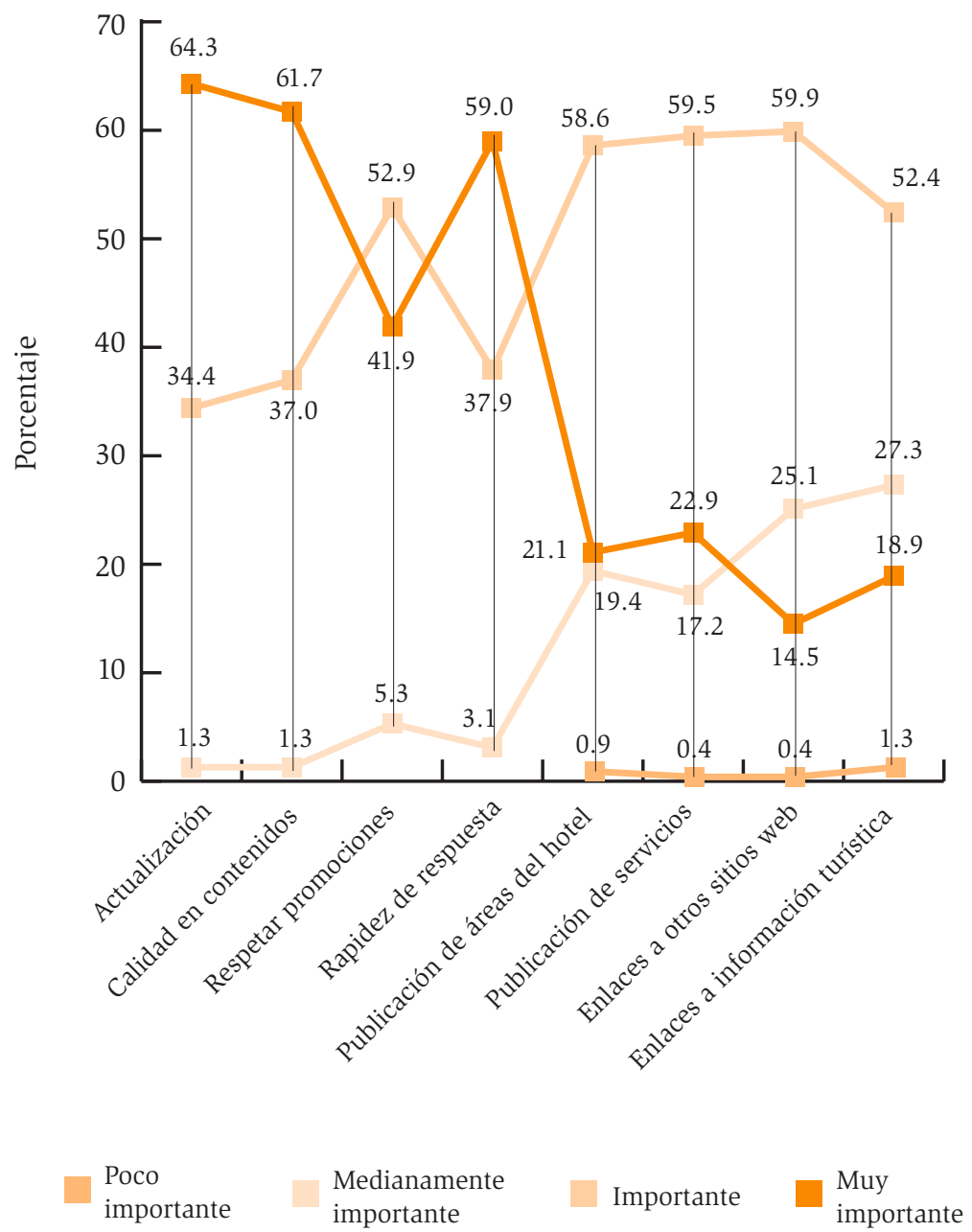

Fuente: Elaboración propia.

Gráfica 7. Factores que aumentan la confianza en la red social del hotel 
fotografías que publiciten los servicios ofertados y anticipen a los turistas la experiencia y satisfacción que tendrán al momento de hospedarse.

Se precisó que $12.5 \%$ de las redes sociales de los hoteles organizan los contenidos por categorías en los espacios dedicados a promocionar los servicios ofertados; tienen en promedio 2.3 años de registro con la red social y el tiempo de respuesta a los mensajes recibidos es en promedio cinco días (dato obtenido únicamente de $31.3 \%$ de los hoteles, debido a que de este porcentaje fue que se obtuvo respuesta).

Además se subraya que la red social más popular entre los huéspedes de los hoteles es Facebook; esto indica que, a pesar de estar presente en otros medios, esta herramienta es la que se usa con mayor frecuencia para acceder a los contenidos publicitados por estas organizaciones en internet.

Esta investigación tiene como finalidad aportar elementos clave para aplicar estrategias de marketing en las redes sociales en los hoteles de cuatro y cinco estrellas localizados en el municipio de Tijuana; a partir de los resultados de este trabajo se reconoce la importancia de las redes sociales como medio de comunicación en la demanda de servicios de hospedaje, así como la asociación entre tales estrategias y la decisión de compra de los clientes; por tal motivo, los empresarios hoteleros deben aprovechar las áreas de oportunidad detectadas y hacer uso de estas herramientas tecnológicas para incrementar su eficiencia en la prestación del servicio haciendo uso de medios electrónicos.

\section{Fuentes consultadas}

AMIPCI (2011). Estudio de comercio electrónico 2011. México: Asociación Mexicana de Internet (AMIPCI) [en línea]. Disponible en: https://www.amipci. org.mx/estudios/comercio_electronico/2011_Comecio_Electronico.pdf [2012, 13 de junio].

(2012). Hábitos de los usuarios de internet en México. México: Amipci [en línea]. Disponible en: https://www.amipci.org.mx/estudios/habitos_ de_internet/2012HabitosUsuariosInternetMx.pdf [2012, 25 de mayo].

Baena, V. (2011). Fundamentos de marketing. Barcelona: Universitat Oberta de Catalunya (Uoc).

Benavides, J. (2012). “De la repetición a la prescripción”. TecnoHotel. Claves 
para los líderes del Turismo [en línea], (451), enero-febrero, 12-15. Disponible en: http://files.epeldano.com/publications/pdf/96/tecnohotel_451.pdf [2012, 25 de mayo].

Casaló, L., C. Flavián y M. Guinalíu (2012). "Redes sociales virtuales desarrolladas por organizaciones empresariales: antecedentes de la intención de participación del consumidor". Cuadernos de Economía y Dirección de la Empresa [en línea], 15 (1), 42-51. Disponible en: http://www. redalyc.org/articulo.oa?id $=80722714005$ [2012, 4 de noviembre].

Colvée, J.L. (coord.) (2007). Estrategias de marketing digital para pymes. Valencia: Anetcom.

Conde, E.M. et al. (2011). Comercialización electrónica en hoteles del pacífico mexicano. Buenos Aires: Elaleph.com.

Domínguez, T. y N. Araújo (2012). "El fenómeno 2.0 en el sector turístico. El caso de Madrid 2.0". PASOS. Revista de Turismo y Patrimonio Cultural [en línea], 10 (3), 225-237. Disponible en: http://www.redalyc.org/ articulo.oa?id $=88123060001$ [2012, 12 de octubre] .

eMarketer (2012). "Is Social Media Marketing at a Saturation Point?” eMarketer [en línea], 17 de agosto. Disponible en: http://www.emarketer.com/ Article.aspx? $\mathrm{R}=1009273$ [2012, 18 de agosto].

Fischer, L. y A.E. Navarro (2000). Introducción a la investigación de mercados. México: McGraw-Hill.

Gallego, J.F. (2008). Marketing para hoteles y restaurantes. Madrid: Paraninfo. García, A.A. (2009). "Redes sociales y aprendizaje a través de las presentaciones on-line”. Teoría de la Educación. Educación y Cultura en la Sociedad de la Información [en línea], 10 (1), 190-216. Disponible en: http://www. redalyc.org/articulo.oa?id $=201018023011$ [2012, 12 de julio].

Hernández, R., C. Fernández y P. Baptista (2006). Metodología de la investigación. México: McGraw Hill.

Hütt, H. (2012). “Las redes sociales: una nueva herramienta de difusión”. Reflexiones [en línea], 91 (2), 121-128. Disponible en: http://www.redalyc.org/articulo.oa?id = 72923962008 [2014, 10 de enero].

Izquierdo, A. y M.P. Martínez (2009). "Análisis de los factores que condicionan la elección del canal de compra por parte del consumidor: evidencias empíricas en la industria hotelera”. Cuadernos de Economía y Dirección 
de la Empresa [en línea], 41, diciembre, 93-122. Disponible en: http:// www.redalyc.org/articulo.oa?id = 80712979004 [2012, 15 de agosto].

Kotler, P. y G. Armstrong (2003). Fundamentos de marketing. México: Pearson Educación.

Laudon, K. y C. Guercio (2009). E-commerce: negocios, tecnología, sociedad. México: Pearson Educación.

Maqueira, J. y S. Bruque (2009). Marketing 2.0 El nuevo marketing en la web de las redes sociales. México: Alfaomega.

Martínez, S.M., J.J. Bernal y J.P. Mellinas (2012). "Los hoteles de la región de Murcia ante las redes sociales y la reputación online”. Revista de Análisis Turístico [en línea], 13, 1-10. Disponible en: http://www.aecit.org/ jornal/index.php/AECIT/article/view/117/108 [2013, 13 de febrero].

Morales, M. (2010). Analítica web para empresas. Arte, ingenio y participación. Barcelona: uoc.

Nielsen (2012). “Confianza en la publicidad”. The Nielsen Company [en línea], 17 de abril. Disponible en: http://www.nielsen.com/us/en/insights/ news/2012/consumer-trust-in-online-social-and-mobile-advertisinggrows.html [2012, 17 de abril].

Pérez, C. (2010). "Marketing con redes sociales”. Suite101 [en línea]. Disponible en: http://suite101.net/article/marketing-con-redes-sociales-a12335 [2012, 13 de julio].

Rojas López, M.D., P. Arango y J.P. Gallego (2009). “Confianza para efectuar compras por Internet”. Dyna [en línea], 76 (160), diciembre, 263-272. Disponible en: http://www.redalyc.org/articulo.oa?id $=49612068017$ [2013, 15 de abril].

Ruiz, C. y S. Sanz (2006). "Influencia de las motivaciones en la decisión de compra y en la lealtad hacia internet". Investigaciones Europeas de Dirección y Economía de la Empresa [en línea], 12 (3), septiembrediciembre, 195-215. Disponible en: http://www.redalyc.org/articulo. oa? id=274120074011 [2013, 30 de enero]. 